\title{
Effects of an anesthesia perioperative surgical home for total knee and hip arthroplasty at a Veterans Affairs Hospital: a quality improvement before-and-after cohort study Effets d'un centre de soins chirurgicaux périopératoires en anesthésie pour les arthroplasties totales du genou et de la hanche dans un hôpital pour anciens combattants : une étude de cohorte d'amélioration de la qualité avant et après
}

\author{
Bret D. Alvis, MD $\mathbb{D} \cdot$ Roland G. Amsler, BBA · Philip J. Leisy, MD • Xiaoke Feng, MS • \\ Matthew S. Shotwell, PhD • Pratik P. Pandharipande, MD • Muhammad Ajmal, MD • \\ Michael McHugh, MD • Ann Walia, MD • Christopher G. Hughes, MD
}

Received: 14 April 2020/Revised: 31 August 2020/Accepted: 15 September 2020/Published online: 2 December 2020

(C) Canadian Anesthesiologists' Society 2020

\begin{abstract}
Background $A$ perioperative surgical home, the Anesthesia Perioperative Care Service (APCS), was created to execute enhanced recovery after surgery
\end{abstract}

Prior presentation Annual Congress of Enhanced Recovery and Perioperative Medicine, 16 March 2018, Fort Lauderdale, FL.

Electronic supplementary material The online version of this article (https://doi.org/10.1007/s12630-020-01865-4) contains supplementary material, which is available to authorized users.

\section{B. D. Alvis, MD ( $\square)$}

Department of Anesthesiology, Vanderbilt University Medical Center, Nashville, TN, USA

e-mail: bret.d.alvis@vumc.org

Department of Anesthesiology, Pain Management \& Perioperative Medicine, Nashville Veterans Affairs Medical Center, Tennessee Valley Healthcare System, Nashville, TN, USA

1211 21st Avenue South, MAB Suite 422, Nashville, TN 37212, USA

R. G. Amsler, BBA

Nashville Veterans Affairs Medical Center, Tennessee Valley Healthcare System, Nashville, TN, USA

P. J. Leisy, MD - P. P. Pandharipande, MD - A. Walia, MD .

C. G. Hughes, MD

Department of Anesthesiology, Vanderbilt University Medical

Center, Nashville, TN, USA pathways for total knee and total hip arthroplasty patients at the Tennessee Valley Health System Nashville VA Medical Center. We hypothesized that the APCS would be associated with reduced length of stay, in-hospital and post-discharge opioid exposure, costs, and hospital readmissions.

Methods Data were collected for all patients admitted to the Nashville VA Medical Center following their respective surgery, for 400 days after the initiation of the APCS and

Department of Anesthesiology, Pain Management \& Perioperative Medicine, Nashville Veterans Affairs Medical Center, Tennessee Valley Healthcare System, Nashville, TN, USA

X. Feng, MS · M. S. Shotwell, PhD

Department of Biostatistics and Department of Anesthesiology,

Vanderbilt University Medical Center, Nashville, TN, USA

M. Ajmal, MD - M. McHugh, MD

Department of Orthopaedic Surgery, Nashville Veterans Affairs Medical Center, Tennessee Valley Healthcare System,

Nashville, TN, USA 
for a 400-day period prior. This cohort study was based on a quality improvement project set up at the initiation of the service. The adjusted effect on each quantitative outcome was evaluated using proportional odds logistic regression methods. In addition, each regression analysis was performed in segmented regression fashion to identify changes in the outcomes over time.

Results We included 282 patients in our cohort-96 prior and 186 post-implementation. Median hospital length of stay, intravenous (IV) and per os (PO) inpatient opioid administration, outpatient opioid quantity, and total days of supply were all reduced in the cohort cared for by the APCS. After adjusting for potential cofounders and evaluated outcome over time, the APCS remained independently associated with a reduction of hospital length of stay of one day (95\% confidence interval, 0.09 to 1.97; $P=0.05$ ) and with decreased IV and PO inpatient opioid administration, while continuing to show no increase in hospital readmissions.

Conclusions This cohort study showed significant improvements in important post-surgical outcomes after total knee and hip arthroplasty that were associated with the implementation of an APCS.

\section{Résumé}

Contexte Un centre de soins chirurgicaux périopératoires (perioperative surgical home), le Service de soins périopératoires en anesthésie (SSPA), a été créé pour mettre en ouvre des trajectoires de soins de récupération rapide après la chirurgie pour les patients ayant subi une arthroplastie totale du genou ou de la hanche au centre médical Tennessee Valley Health System Nashville VA Medical Center. Nous avons émis l'hypothèse que le SSPA serait associé à une réduction de la durée du séjour, de l'exposition aux opiö̈les à l'hôpital et après le congé, ainsi qu'à une diminution des coûts et des réadmissions à l'hôpital.

Méthode Les données ont été recueillies pour tous les patients admis au centre médical Nashville VA Medical Center après leur chirurgie respective, pendant 400 jours avant et après la création du SSPA. Cette étude de cohorte se fondait sur un projet d'amélioration de la qualité mis en place lors de l'inauguration du service. L'effet ajusté sur chaque résultat quantitatif a été évalué à l'aide de méthodes de régression logistique proportionnelles. De plus, chaque analyse de régression a été effectuée de façon segmentée afin d'identifier l'évolution des résultats au fil du temps.

Résultats Nous avons inclus 282 patients dans notre cohorte - 96 avant et 186 après la mise en ouvre. La durée médiane du séjour à l'hôpital, l'administration d'opioïles par voie intraveineuse (IV) et per os (PO) pendant le séjour hospitalier, la quantité d'opioïdes en ambulatoire et sa durée en jours ont tous été réduites dans la cohorte prise en charge par le SSPA. Après avoir procédé à des ajustements pour tenir compte des facteurs de confusion potentiels et évalué l'évolution des résultats au fil du temps, le SSPA est demeuré indépendamment associé à une réduction de la durée de séjour à l'hôpital d'un jour (intervalle de confiance $95 \%, 0,09$ à 1,97; $P=$ 0,05), à une réduction de l'administration d'opioïdes IV et PO durant le séjour, et il n'y a eu aucune augmentation des réadmissions à l'hôpital.

Conclusion Cette étude de cohorte a montré des améliorations significatives en matière de résultats postchirurgicaux importants après une arthroplastie totale $d u$ genou et de la hanche associés à la mise en ouvre d'un SSPA.

Keywords perioperative $\cdot$ surgical home enhanced recovery after surgery $\cdot$ ERAS · opioids

The perioperative surgical home (PSH) is a patient-centred multidisciplinary approach to comprehensive perioperative management, adopted by the American Society of Anesthesiologists, to improve the quality of patient care and increase safety. ${ }^{1-3}$ The PSH model promotes shared decision-making and has been successfully implemented in hospitals throughout the United States. ${ }^{1,2,4,5}$ The Institute for Healthcare Improvement promotes the "Triple Aim" as the backbone to healthcare reform to help contain spending and improve outcomes. ${ }^{6}$ These three aims are: 1) improve individual experience of care, 2) improve the health of populations, and 3) reduce per capita costs of care. ${ }^{6}$ To achieve these goals, the PSH has been supported. The PSH has a strong emphasis on standardization, coordination, transitions, and value of care throughout the perioperative continuum, including post-discharge. ${ }^{6}$ To date, however, there remains uncertainty regarding how to optimally structure a PSH model and whether a PSH model improves patient outcomes. One possible effective method is implementing enhanced recovery after surgery (ERAS) pathways into the heart of the PSH. Enhanced recovery after surgery pathways are evidence-based multimodal interventions that help to achieve early postoperative recovery. ${ }^{5,7}$ These pathways focus interventions on common reasons for prolonged hospitalization after surgery and commonly involve several members of the perioperative care team. ${ }^{5,8}$ There is abundant clinical evidence supporting improved outcomes with the use of ERAS pathways. ${ }^{5}$

A PSH model implementing ERAS pathways has the potential to decrease length of stay, inpatient opioid use, cost, and hospital readmissions; but there are limited data 
evaluating these outcomes. Further, a PSH model may help stem the increasingly adverse impact of postoperative misuse and abuse of opioids in the United States. ${ }^{9}$ Opioid use in the United States is endemic and increasing, ${ }^{10}$ and opioid abuse is especially prevalent in the veterans' health administration population, with rates almost seven times higher than in the general population. ${ }^{11}$ Baser et al. showed that the total healthcare cost burden of opioid users is four times higher in veterans than in patients without prescription opioids, ${ }^{11}$ and opioid use greater than 90-days has been reported as high as $18.3 \%$ in veterans. ${ }^{12}$ Veterans are at risk for prolonged opioid use following surgery, including total knee arthroplasty (TKA). ${ }^{13,14}$ Whether a PSH at a Veterans Affairs Hospital can reduce chronic opioid use after surgery is not known. Nevertheless, it is well known that more work needs to be done to improve and reduce the quantity, dosages, days of supply, and chronic opioid use in our veterans.

We have previously described the implementation of an Anesthesia Perioperative Care Service (APCS) at a Veterans Affairs Hospital that combines a PSH model with ERAS pathways. ${ }^{1}$ The service was created with input from representatives from surgery, anesthesiology, nursing, critical care, social work, pharmacy, and physical therapy. At our hospital, no ERAS pathways were used and a PSH concept was not in place for any surgical patient prior to the initiation of the APCS. We hypothesized that the APCS would be associated with reduced length of stay, inhospital and post-discharge opioid exposure, costs, and readmissions. To test these hypotheses, we performed a cohort study of patients undergoing TKA and total hip arthroplasty (THA), including patients two years prior to APCS implementation and up to two years after APCS implementation.

\section{Methods}

Study design and population

The service's data collection and analysis were reviewed and approved by the Tennessee Valley Healthcare System Nashville VA Institutional Review Board (IRB). The project was deemed quality improvement and the requirement for written informed consent for this quality improvement project was waived by the IRB at the initiation of this service. The setting for this project was described in detail in an earlier publication. ${ }^{1}$ At the Tennessee Valley Healthcare System Nashville VA Medical Center, the Department of Anesthesiology, Pain Management \& Perioperative Medicine developed an APCS to provide $24 / 7$ care for our surgical patients. ${ }^{1}$
ERAS pathway execution for all TKA and THA patients within the APCS was initiated on 1 January 2016. Data were collected for patients admitted to the Nashville VA Medical Center for 400 days prior to APCS (no ERAS/ PSH) and 400 days after the initiation of APCS for TKA and THA surgical procedures (Fig. 1). Each subject's VA outpatient opioid prescription fills and VA hospital readmission data were collected for 180 days after their surgical discharge date.

\section{Exposures}

Our APCS is a PSH model performing ERAS pathways led by critical care anesthesiologists. ${ }^{1}$ Staffing includes fulltime APCS-dedicated nurse practitioners. ${ }^{1}$ All postsurgical patients on ERAS pathways are cared for by the APCS in conjunction with the surgical team. A detailed description of this care service can be found in our previous publication. ${ }^{1}$ The independent variable for this study was the involvement of the APCS in the perioperative patient care, as determined by the date of the surgical admission prior to or after the APCS initiation. No other significant care pattern changes occurred in the surgical pathway of TKA and THA. The analgesia specifics for the TKA/THA ERAS pathway included a preoperative dose of acetaminophen, meloxicam, and gabapentin, in addition to intraoperative periarticular joint injection of ropivacaine, epinephrine, and ketorolac. Postoperative analgesia was provided with scheduled acetaminophen, gabapentin, and meloxicam and oxycodone as needed. Intravenous (IV) opioids (either hydromorphone or morphine) were ordered as needed and provided to the patient at the discretion of the bedside nurse based on pain assessments. The decision to place patients on an IV opioid patient-controlled analgesia (PCA) infusion was the responsibility of the APCS team. The ERAS pathway protocolized other aspects of care such as postoperative nausea/vomiting prophylaxis and treatment, temperature control, fluid administration, rehabilitation, medicine reconciliation, social work followup, discharge criteria, and follow-up.

\section{Outcomes}

The dependent variables for this study included length of stay, in-hospital and post-discharge opioid exposure, total hospital cost, and hospital readmission. Length of stay was determined in days, from the day of admission for the surgical procedure until the actual day of discharge to home or to an institution (not when the patient was deemed medically ready for discharge). Inpatient opioid exposure was determined as total IV opioid administration in morphine equivalents (ME) and as total oral opioid administration in ME. This did not include opioids 


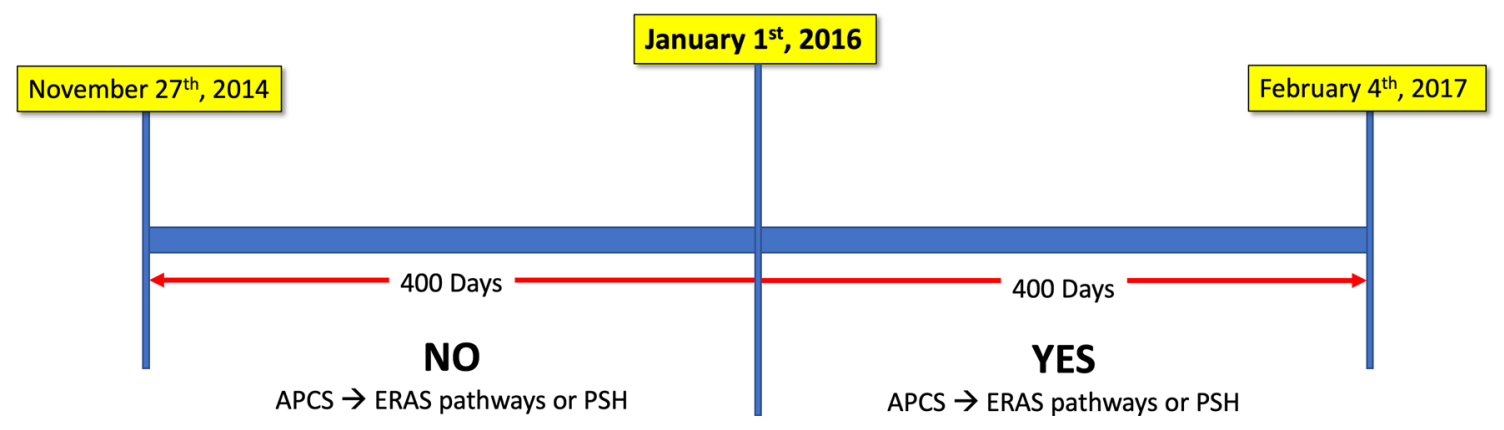

Fig. 1 Timeline of data collection before and after the initiation of the Anesthesia Perioperative Care Service. Data were collected for patients admitted to the Nashville VA Medical Center for 400 days before initiation of the APCS and 400 days after initiation of the APCS for patients undergoing TKA and THA surgical procedures.

administered intraoperatively. Post-discharge opioid exposure was determined by prescription dosage filled in ME (mg), prescription quantity (\# pills) filled, and prescription days of supply. Chronic opioid use was determined by any patient who continued to receive and fill their opioid prescription beyond six months from postprocedure discharge. ${ }^{15}$ The total cost was determined by the sum of the surgical procedure cost and the cost of inpatient medical care, including medications, professional coverage costs, therapy costs, etc. Thirty-day readmission was determined as admitted to a VA hospital within 30-days after their surgical discharge date, regardless of reason.

\section{Covariates}

We chose potential confounders of the associations between APCS and outcomes a priori based on previous research and clinical judgement. We focused primarily on baseline variables that would potentially impact patient responses to our pathway medications, pain scores, opioid requirements, and overall hospital course. These included age, sex, body mass index (BMI), American Society of Anesthesiologists (ASA) classification, chronic opioid use within six months prior to surgery (yes $v s$ no), and type of surgery (TKA $v s$ THA).

\section{Statistical analysis}

Patient demographics and clinical characteristics were summarized using the median and interquartile range for continuous variables and percentages for categorical variables. The Wilcoxon rank sum test and the Pearson Chi square test were performed as appropriate for comparisons between service types $($ APCS $=$ yes $v s$ APCS $=$ no).
Follow-up for these subjects lasted an additional 180 days for both subsets of subjects to evaluate post-discharge outcomes such as outpatient medication use and readmissions. APCS = Anesthesia Perioperative Care Service; ERAS = enhanced recovery after surgery; THA = total hip arthroplasty; TKA = total knee arthroplasty

The adjusted effect of APCS on each quantitative outcome was evaluated using proportional odds logistic regression methods. This technique permitted us to adequately account for boundedness, skew, and heteroscedasticity in outcomes (e.g., IV opioid ME), and is suitable for ordinal and continuous outcomes. ${ }^{16}$ Logistic regression was used to evaluate the effect of APCS on binary outcomes. All regression analyses were adjusted for age, sex, ASA classification, BMI, opioid use six months prior to surgery, and surgery type (TKA vs THA). Age and BMI were modelled using a four-knot natural cubic spline to allow for nonlinear associations. In addition, because APCS was implemented in a pre-post fashion, each regression analysis was performed in a segmented regression fashion, which quantifies both the change in average outcome at the time of implementation and the change in outcome trend. The segmented regression method is used to identify changes in the outcome over time that may confound the association between APCS and outcomes. ${ }^{17}$ The overall effect of APCS on each outcome is summarized by testing (Wald-type) the null hypothesis that APCS had no effect on either the average outcome or the outcome trend. In addition, the effect of APCS on each outcome is summarized graphically and by comparing the mean outcomes at 1) the beginning of the observation period, 2) just before APCS implementation, 3) just after APCS implementation, and 4) at the end of the observation period.

All analyses were implemented using R 3.3.0 ( $\mathrm{R}$ Foundation for Statistical Computing, Vienna, Austria). A significance level of 0.05 was used for statistical inference.

\section{Results}

Our cohort included 282 patients, 186 of whom were cared for by the APCS and 96 of whom were cared for before the 
(A)
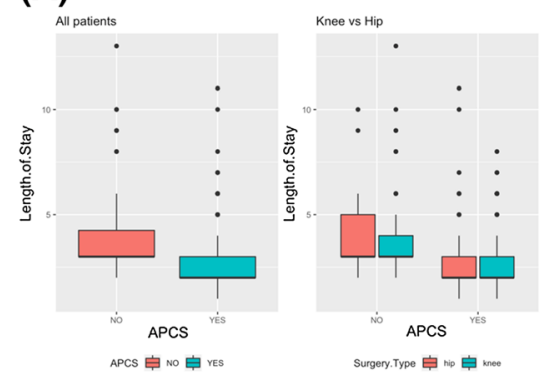

(D)

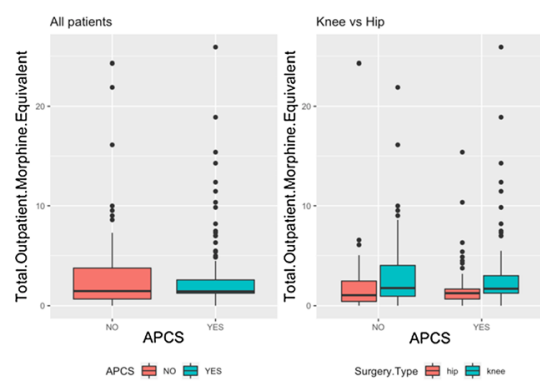

(B)

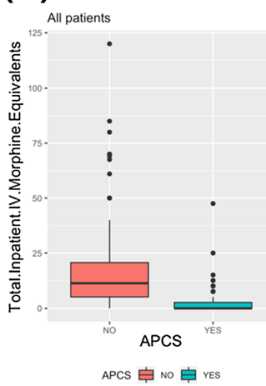

(E)

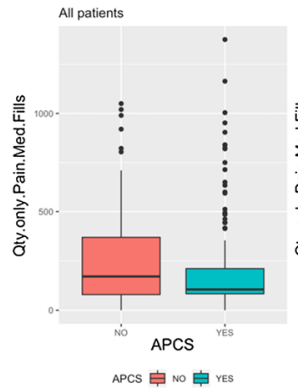

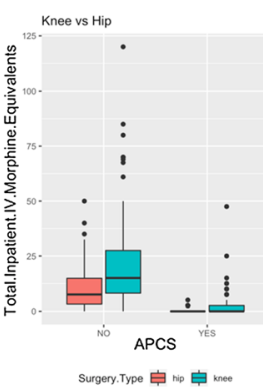

Surgery.Type 自 hip 自 knoo

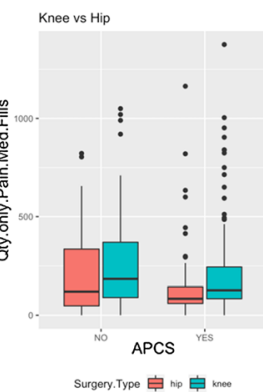

(C)
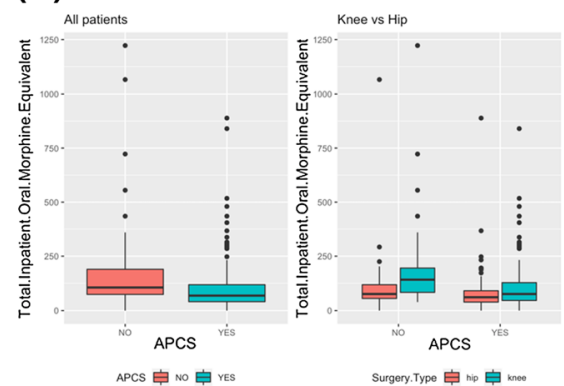

(F)

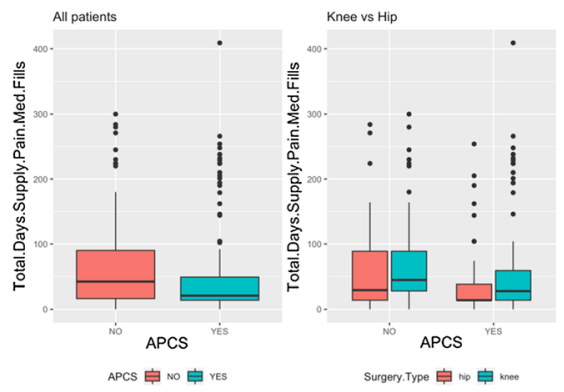

Fig. 2 Outcome data plots representing 400 days before initiation of the Anesthesia Perioperative Care Service (NO) vs 400 days after initiation of the Anesthesia Perioperative Care Service (YES). Median with IQR of length of stay (A), total inpatient IV morphine equivalents (B), total inpatient oral morphine equivalents (C), total outpatient morphine equivalents (D), quantity of pain medications filled (E), and total days of supply (F). Hip = total hip arthroplasty, $\mathrm{IQR}=$ interquartile range; IV = intravenous; Knee = total knee arthroplasty

was a significant immediate effect of APCS on inpatient IV opioid intake in ME that persisted over time (ESM, eFig. 1). After APCS initiation, the median inpatient IV opioid intake in ME was decreased by $7.48 \mathrm{mg}$ (95\% CI, 1.73 to 13.24$)$ for THA patients and by $11.48 \mathrm{mg}$ (95\% CI, 3.34 to 19.62) for TKA patients. In the adjusted analysis, the APCS service was independently associated with a reduction of inpatient IV opioid intake (ESM eTable).

Median [IQR] inpatient oral opioid intake in ME for all patients was 68 [39-118] $\mathrm{mg}$ in the implementation phase compared with 105.0 [73-189] $\mathrm{mg}$ before the implementation phase (Table, Fig. 2). There was a significant effect of APCS on both inpatient oral opioid intake in ME and trend over time (ESM eFig. 1). For both THA and TKA patients, the average inpatient oral opioid intake in ME was increasing before the APCS was initiated. In the implementation phase, the average inpatient oral opioid intake in ME decreased by 82.05 mg (95\% CI, 25.94 to 138.16) for THA patients and by $117.28 \mathrm{mg}$ (95\% CI, 38.33 to 196.22) for TKA patients. The average inpatient oral opioid intake in ME remained stable after the intervention. After adjusting for confounders, the APCS service was independently associated with a decrease in inpatient oral opioid intake in ME (ESM eTable). 
TABLE Demographics and outcomes for patients prior to initiation of APCS compared with patients after the initiation of the APCS

\begin{tabular}{|c|c|c|c|c|}
\hline Demographics and Outcomes & $\begin{array}{l}\text { Pre-APCS } \\
n=96\end{array}$ & $\begin{array}{l}\text { Post-APCS } \\
n=186\end{array}$ & Effect size $(95 \% \mathrm{CI})$ & $P$ value \\
\hline \multirow[t]{2}{*}{ Age (yr) } & $65.0[60.8-69.2]$ & $63.5[58.2-68.0]$ & & $P=0.11^{1}$ \\
\hline & $64.8(9.0)$ & $62.9(8.3)$ & & \\
\hline Sex & & & & $P=0.30^{2}$ \\
\hline Female & $12(12 \%)$ & $16(9 \%)$ & & \\
\hline Male & $84(88 \%)$ & $170(91 \%)$ & & \\
\hline ASA & & & & $P=0.21^{2}$ \\
\hline II & $11(11 \%)$ & $36(19 \%)$ & & \\
\hline III & $81(84 \%)$ & $145(78 \%)$ & & \\
\hline IV & $4(4 \%)$ & $5(3 \%)$ & & \\
\hline \multirow[t]{2}{*}{ BMI } & $30.9[27.2-34.3]$ & $30.9[27.1-34.4]$ & & $P=1.00^{1}$ \\
\hline & $30.8(5.4)$ & $30.7(5.2)$ & & \\
\hline Opioid within 6 months & $39(41 \%)$ & $79(42 \%)$ & & $P=0.77^{2}$ \\
\hline Surgery type & & & & $P=0.66^{2}$ \\
\hline Hip & $34(35 \%)$ & $61(33 \%)$ & & \\
\hline Knee & $62(65 \%)$ & $125(67 \%)$ & & \\
\hline \multicolumn{5}{|l|}{ Outcomes } \\
\hline \multirow[t]{2}{*}{ Length of stay (days) } & $3.0[3.0-4.2]$ & $2.0[2.0-3.0]$ & $1.1(0.6$ to 1.6$)$ & $P<0.001^{1}$ \\
\hline & $3.9(2.1)$ & $2.8(1.5)$ & & \\
\hline \multirow[t]{2}{*}{ Inpatient IV morphine equivalents } & $11.2[5.0-20.6]$ & $0.0[0.0-2.5]$ & 17.3 (12.9 to 21.8$)$ & $P<0.001^{1}$ \\
\hline & $18.9(21.9)$ & $1.6(4.6)$ & & \\
\hline \multirow[t]{2}{*}{ Total inpatient oral morphine equivalent } & 105 [73-189] & 68 [39-118] & $54(14$ to 95$)$ & $P<0.001^{1}$ \\
\hline & $158(182)$ & $104(119)$ & & \\
\hline \multirow[t]{2}{*}{ Outpatient morphine equivalent } & $1,472[675-3,742]$ & $1,440[1,260-2,555]$ & $462(-464$ to 1389$)$ & $P=0.83^{1}$ \\
\hline & $2,927(3,977)$ & $2,465(3,211)$ & & \\
\hline \multirow[t]{2}{*}{ Total outpatient opioid quantity filled (number of pills) } & $170[80-369]$ & $106[84-210]$ & $68(7$ to 129$)$ & $P=0.02^{1}$ \\
\hline & $262(253)$ & $195(228)$ & & \\
\hline \multirow[t]{2}{*}{ Total outpatient opioid days of supply (days) } & $42[16-90]$ & 21 [14-49] & $20(2$ to 38$)$ & $P<0.001^{1}$ \\
\hline & $70(74)$ & $51(68)$ & & \\
\hline Chronic opioid use & $17(18 \%)$ & $25(13 \%)$ & $1.32(0.75$ to 2.32$)$ & $P=0.34^{2}$ \\
\hline \multirow[t]{2}{*}{ Total cost (USD) } & $7,150[5,559-14,624]$ & $10,716[7,765-29,185]$ & $-6,747(-9,696$ to $-3,798)$ & $P<0.001^{1}$ \\
\hline & $11,894(9,817)$ & $18,641(15,183)$ & & \\
\hline Readmission & $19(20 \%)$ & $25(13 \%)$ & $1.47(0.86,2.54)$ & $P=0.16^{2}$ \\
\hline
\end{tabular}

Continuous variables are median [IQR] and mean (SD). ${ }^{1}$ Wilcoxon test and ${ }^{2}$ Pearson test were used to make unadjusted comparisons for continuous and categorical variables, respectively. Difference in means and 95\% CI were calculated for continuous outcomes, risk ratio and 95\% CI were calculated for binary outcomes

ASA = American Society of Anesthesiologists; APSC = Anesthesia Perioperative Care Service; $\mathrm{BMI}=$ body mass index; $\mathrm{CI}=$ confidence interval; IV = intravenous

To evaluate the effect of the APCS on outpatient opioid administration, median $[\mathrm{IQR}]$ total outpatient opioid dosages, amounts, and days of supply were evaluated. The median [IQR] total outpatient opioid dosage filled at a VA pharmacy expressed in ME for all patients was 1,440 [1,260-2,555] $\mathrm{mg}$ after APCS compared with 1,472 [675-3,742] $\mathrm{mg}$ before APCS (Table, Fig. 2). In the adjusted analysis, there was no evidence showing association between the APCS service and outpatient opioid dosage filled at a VA pharmacy expressed in ME (ESM eTable).

The median [IQR] total outpatient opioid quantity filled expressed in number of pills in all patients was significantly lower at 106 [84-210] pills after APCS compared with 170 [80-369] pills before APCS (Table, Fig. 2). After adjusting for potential confounders, the APCS was not significantly associated with reduction in 
outpatient opioid pills filled at a VA pharmacy (ESM eTable).

The median [IQR] total outpatient opioid days of supply for all patients was significantly lower at 21 [14-49] days with the APCS compared with 42 [16-90] days without the APCS (Table, Fig. 2). After adjusting for potential confounders, there was no significant association of APCS with total outpatient opioid days of supply (ESM eTable).

In all patients, the percentage of chronic opioid use was $13 \%$ after APCS implementation compared with $18 \%$ before APCS implementation (Table, ESM eFig. 2). In the adjusted analysis, there was no significant association between the APCS service and chronic opioid use (ESM eTable).

\section{Cost}

The median cost for patients was USD 7,150 for those not cared for by the APCS compared with USD 10,716 for patients cared for by the APCS (Table). In the adjusted analysis, APCS service was not independently associated with reduced total cost (ESM eTable).

\section{Readmission}

The percentage all-cause 30-day readmissions was $13 \%$ after the APCS was implemented compared with $20 \%$ before the APCS was implemented (Table, ESM eFig. 2). The APCS service was not significantly associated with reduced readmission after adjusting for potential confounders (ESM eTable).

\section{Discussion}

In this cohort study, we found that the APCS was independently associated with a decrease in length of stay and inpatient opioid exposure. Outpatient opioid exposure, chronic opioid use, cost, and readmission were also significantly lower after APCS; however, we did not identify an independent association between the APCS and these outcomes when adjusted for potential cofounders. Our data indicate that a dedicated APCS team led by critical care anesthesiologists and staffed by nurse practitioners is independently associated with improved hospital throughput and less opioid exposure while also having the potential to reduce long-term opioid exposure, cost, and readmission.

Over the past three years, we have successfully implemented a PSH model at the Tennessee Valley Healthcare System-VA, with critical care anesthesiologists expanding their services to cover post- surgical ward patients. This, along with full-time APCSdedicated nurse practitioners, has allowed us to achieve a successful fusion of the PSH with ERAS pathways. The department of anesthesia routinely reports on the APCS outcomes to the hospital leadership, anesthesiologists, and surgeons, as well as the nurses and nurse anesthetists. This routine feedback has been imperative to the necessary "buy-in" from these important parties outside of the APCS team. As Mariano et al. explain, a well-documented challenge with implementing enhanced recovery programs is that adherence decreases as protocols are developed. ${ }^{4}$ To support adherence to ERAS, our APCS team, as described previously, ${ }^{1}$ follows each TKA and THA patient from the time the surgery is scheduled to seven days post-discharge. While they are in the hospital, they are followed by one of eight registered nurse practitioners and staffed by one full-time critical care anesthesiologist 24/7. This aggressive staffing model was important from the first day given the expected variability in adherence, and it allowed for constant feedback from providers and patients, evaluation and monitoring of adherence to the ERAS pathways, and constant support of pain management. To date, however, it was unclear if this program, while feasible, was associated with improvement in outcomes and how this increased staffing affected hospital costs. This work expands on our previous report ${ }^{1}$ that described non-billable work and justification for the resources being allocated to this initiative by investigating the outcomes and cost saving goals.

When comparing the median length of stay in days from the 400 days before initiation of the APCS to the 400 days after implementation, a full day out of the hospital was gained. Using the Health Economics Resource Center inpatient data on the 2014 average VA surgical inpatient daily cost (USD 5,121 per day), ${ }^{15}$ an estimated USD 952,506 has been saved based on reduced length of stay. When the average total surgical costs were pulled from the Managerial Cost Accounting System Surgical National Data Extract for our patient cohorts, there was an increase in operative cost range of TKA of USD 341-USD 2,972 and THA of USD 1,725-USD 2,256 after APCS implementation. Therefore, even at the top end of the ranges (USD 2,972 and USD 2,256, respectively), this increase in total operative cost for the patient totaled USD 509,116. Even if all of this increased cost was attributed to the intraoperative ERAS protocols (and not to pricing changes for equipment, etc.), an estimated net saving of USD 443,390 was shown, as reduced length of stay savings were greater than increased costs. When a more granular evaluation of the total cost at the level of each individual patient was analyzed using a multivariable regression, the results showed that, despite lower average costs, the APCS team was not independently associated with reduced 
overall cost in multivariable models. Of note, the shorter stay did not increase the 30-day all-cause readmissions. In fact, while not statistically significant, readmissions were lower post-intervention. This is a significant finding given that patients are going home a day sooner. One of the first concerns with the initiation of the APCS was that TKA and THA patients would be "going home" too early and would end up being readmitted. Nevertheless, this was not witnessed.

Our APCS also aimed to decrease opioid use in the hospital as well as the outpatient burden. This was a major goal of the ERAS pathway, and why "multimodal analgesia" was a point of emphasis within both the TKA and THA pathway. The dosage of IV opioids used for both TKA and THA was significantly reduced with the initiation of the ERAS pathways and the APCS. Total knee arthroplasty and THA patients decreased from a median of $15 \mathrm{mg}$ and $7.5 \mathrm{mg}$ of IV ME to $0.0 \mathrm{mg}$ and $0.0 \mathrm{mg}$ of IV ME, respectively. While its use was not restricted by the ERAS pathways, the opioid PCA was not ever required after APCS initiation. A significant reduction in inpatient oral ME was also seen for TKA with a decrease from 141 $\mathrm{mg}$ to $75 \mathrm{mg}$. For THA patients, after the initiation of the APCS, inpatient oral ME decreased from $75 \mathrm{mg}$ to $60 \mathrm{mg}$, but this reduction was not significant. This decrease in opioid intake may have reduced the length of stay secondary to reducing their well-documented negative side effects (e.g., nausea, emesis, urinary retention, ileus) and was likely, also, a contributing reason to the additional savings of the APCS despite the higher operative costs of each patient. We further investigated the quantity of opioid use outside the hospital. The multitude of variables that affect the opioid dosage used for pain control led us to evaluate outpatient opioid dosages, amounts (\# pills), and days of supply and whether patients continued to use opioids six months after the operation. The number of pills was chosen as a specific outcome to appreciate the large variance in dosing need that comes with each individual patient and to also investigate the sheer number of opioid quantities making its way out into society. Both TKA and THA patients had on average 67 less pills after the APCS was initiated. When this was evaluated in ME, the dosage filled was approximately $462 \mathrm{mg}$ less for all patients. The patients included after APCS involvement also showed a reduction of their average days of opioid supply by 19 days. Despite these differences in prescribing, we did not find a statistically significant effect of the APCS on postdischarge prescriptions. While the variables that account for a patient's dosing strategy are complex, patients had less chronic ( $>$ six months) opioid use (13\% vs 18\%) after APCS implementation; however, there was not a significant independent association in multivariable analyses.
This is one of the first studies to assess whether PSH models can improve outcomes and drive changes in healthcare cost. The sample size and data collection allowed us to test the independent association of the service intervention while adjusting for important potential confounders and the effect of time. We explored the important outcome of hospital throughput, inpatient and outpatient opioid exposure, cost, and readmission. The VA medical system allowed us to track prescriptions and admissions across the enterprise in a patient population that predominantly uses this single system for care, giving us more inclusive data than for many other populations. Future projects could also include reporting quantitative and qualitative feedback from patients; this would allow rich analysis of themes that are a priority for both the PSH and ERAS pathways and any future quality improvement studies.

There were several limitations to our analysis. ERAS pathways were an integral component to our PSH model, with both simultaneously occurring; thus, determining if benefits were secondary to the ERAS pathway $v s$ the $\mathrm{PSH}$ was not possible. We agree with King et al. ${ }^{5}$ that the fusion of the two is the ideal clinical condition, so separating the two for analysis was not deemed appropriate. To track outpatient opioid use/dosages, we assumed that each patient was only filling VA prescriptions and consuming only the opioids they received from the VA. Also, the APCS made outpatient opioid recommendations to the primary surgeon, but it was the surgeon's responsibility to treat outpatient pain and oversee outpatient opioid use/consumption. This limitation was true for readmissions as well. Only readmissions to the VA were captured. Therefore, it could be under-represented in our data set. We could only track overall hospital costs for each patient and not costs directly related to the APCS service. Furthermore, this is a single-centre study in a VA medical centre, which limits generalizability. Intraoperative opioid administration could not be investigated in our data set secondary to the current charting practice of pen and paper. Finally, this pre-post cohort study could not test causation, and the results could have been affected by unknown confounding. Though we were able to account for several potential confounders and the effect of time in our statistical analyses, additional aspects such as baseline frailty, surgical technique and wound care, and adherence to specific ERAS bundle components (e.g., intraoperative drug exposure, postoperative physical therapy) were not accounted for. 


\section{Conclusion}

Our fusion of the PSH with ERAS pathways, the APCS, showed a reduction in length of stay, total inpatient IV and oral opioid intake, quantity of outpatient oral opioid, and total days of opioid supply. These are major benefits to the veterans at our local site and may be relevant at other sites. Nevertheless, more work needs to be done to further reduce the quantity, dosages, days of supply, and chronic nature of opioid use. The Tennessee Valley Healthcare SystemVeterans Affairs Hospital APCS continues to care for our veterans to this day with the same motivation and commitment to improving the quality of care. Further study of this type of program in community and academic centres is also warranted.

Acknowledgements The authors would like to thank each of the nurse practitioners working on the Anesthesia Perioperative Care Service for their hard work and dedication: Katelin Avila, Misty Tuck, Daniel Miser, Anika Boyd, Mahogany Anthony, Molly Goidel, Sopheap Phathanak, Melissa Wiley, and Samantha Boyd. It is with their hard work that these outcomes are possible.

Author contributions Bret D. Alvis, Roland G. Amsler, Philip J. Leisy, Pratik P. Pandharipande, Muhammad Ajmal, Michael McHugh, Ann Walia, and Christopher G. Hughes performed study design, data analysis, manuscript preparation, and manuscript revisions. Bret D. Alvis, Roland G. Amsler, Xiaoke Feng, and Matthew S. Shotwell analyzed the data. Bret D. Alvis, Xiaoke Feng, and Matthew S. Shotwell prepared the figures. Bret D. Alvis had full access to all of the data in the study, takes responsibility for the integrity of the data and the accuracy of the data analysis, and had final responsibility for the decision to submit for publication.

Conflict of interest Human data were recorded per standard VA procedures and investigated as part of an Institutional Review Boardapproved quality improvement project set up at the initiation of the Anesthesia Perioperative Care Service. The Institutional Review Board waived the need for consent.

\section{Disclosures None.}

Funding statement This research did not receive any specific grant from funding agencies in the public, commercial, or not-for-profit sectors.

Editorial responsibility This submission was handled by Dr. Philip M. Jones, Associate Editor, Canadian Journal of Anesthesia.

\section{References}

1. Alvis BD, King AB, Pandharipande PP, et al. Creation and execution of a novel Anesthesia Perioperative Care Service at a Veterans Affairs Hospital. Anesth Analg 2017; 125: 1526-31.
2. King AB, McEvoy MD, Fowler LC, et al. Disruptive education: training the future generation of perioperative physicians. Anesthesiology 2016; 125: 266-8.

3. Dexter F, Wachtel RE. Strategies for net cost reductions with the expanded role and expertise of anesthesiologists in the perioperative surgical home. Anesth Analg 2014; 118: 1062-71.

4. Mariano ER, Vetter TR, Kain ZN. The perioperative surgical home is not just a name. Anesth Analg 2017; 125: 1443-5.

5. King AB, Alvis BD, McEvoy MD. Enhanced recovery after surgery, perioperative medicine, and the perioperative surgical home: current state and future implications for education and training. Curr Opin Anaesthesiol 2016; 29: 727-32.

6. Vetter TR, Boudreaux AM, Jones KA, Hunter JM Jr, Pittet JF. The perioperative surgical home: how anesthesiology can collaboratively achieve and leverage the triple aim in health care. Anesth Analg 2014; 118: 1131-6.

7. McEvoy $M D$, Wanderer $J P$, King $A B$, et al. A perioperative consult service results in reduction in cost and length of stay for colorectal surgical patients: evidence from a healthcare redesign project. Perioper Med (Lond) 2016; DOI: https://doi.org/10.1186/ s13741-016-0028-1.

8. Scott MJ, Baldini G, Fearon KC, et al. Enhanced Recovery After Surgery (ERAS) for gastrointestinal surgery, part 1: pathophysiological considerations. Acta Anaesthesiol Scand 2015; 59: 1212-31.

9. Vetter TR, Kain ZN. Role of the perioperative surgical home in optimizing the perioperative use of opioids. Anesth Analg 2017; 125: $1653-7$.

10. Manchikanti L, Helm $S 2^{\text {nd }}$, Fellows B, et al. Opioid epidemic in the United States. Pain Physician 2012; 15(3 Suppl): ES9-38.

11. Baser O, Xie L, Mardekian J, Schaaf D, Wang L, Joshi AV. Prevalence of diagnosed opioid abuse and its economic burden in the veterans health administration. Pain Pract 2014; 14: 437-45.

12. Mosher HJ, Richardson KK, Lund BC. The 1-year treatment course of new opioid recipients in veterans health administration. Pain Med 2016; 17: 1282-91.

13. Ben-Ari A, Chansky H, Rozet I. Preoperative opioid use is associated with early revision after total knee arthroplasty: a study of male patients treated in the Veterans Affairs System. J Bone Joint Surg Am 2017; 99: 1-9.

14. Hadlandsmyth K, Vander Weg MW, McCoy KD, Mosher HJ, Vaughan-Sarrazin MS, Lund BC. Risk for prolonged opioid use following total knee arthroplasty in veterans. J Arthroplasty 2018; 33: 119-23.

15. Hah JM, Bateman BT, Ratliff J, Curtin C, Sun E. Chronic opioid use after surgery: implications for perioperative management in the face of the opioid epidemic. Anesth Analg 2017; 125: 1733-40.

16. Liu $Q$, Shepherd BE, Li C, Harrell FE Jr. Modeling continuous response variables using ordinal regression. Stat Med 2017; 36: 4316-35.

17. Mascha EJ, Sessler DI. Segmented regression and difference-indifference methods: assessing the impact of systemic changes in health care. Anesth Analg 2019; 129: 618-33.

Publisher's Note Springer Nature remains neutral with regard to jurisdictional claims in published maps and institutional affiliations. 